\title{
Novelty without toxicity: a quest for a safer local anesthetic
}

\author{
Vivian Hui Yun Ip, MB • Ban C. H. Tsui, MD
}

Received: 24 September 2010/Accepted: 14 October 2010/Published online: 2 November 2010

(c) Canadian Anesthesiologists' Society 2010

The search for an "ideal" local anesthetic for perioperative anesthesia and analgesia has continued for more than a century. The ideal local anesthetic is an agent with a rapid onset and desirable duration of action that is capable of producing reversible and selective blockade of sensory nerve conduction without risks of local (neurotoxic and myotoxic) or systemic (neurological and cardiovascular) toxicity. Since Koller's introduction of cocaine as a surgical anesthetic in 1884, several hundred compounds have been tested and found to produce local anesthetic effects. The chemical structures of these agents are diverse and include amino esters (procaine analogues), aminoamides (lidocaine analogues), alcohols (benzyl alcohol), and phenol. However, only a handful of these agents are of clinical value, and the search for the "ideal" local anesthetic continues.

For decades, systemic local anesthetic toxicity has received much attention due to the relatively large quantity of local anesthetic administered when performing regional anesthesia. Given the advent of ultrasound-guided regional anesthesia, it is now possible to deposit local anesthetic in close proximity to the desired neurological structure with great precision. Therefore, most regional anesthesia experts are capable of producing successful and desirable effects using substantially reduced quantities of local anesthetic. Furthermore, the ability to visualize the anatomic surroundings in a dynamic manner allows the spread of local anesthetic to take place with a greatly reduced risk of accidental intravascular injection. Although the evidence is

V. H. Y. Ip, MB · B. C. H. Tsui, MD (ه)

Department of Anesthesiology and Pain Medicine, University of Alberta Hospital, 8-120 Clinical Sciences Building, Edmonton, AB T6G 2G3, Canada

e-mail: btsui@ualberta.ca still pending, it is likely that this advance in technology holds great promise for reducing the incidence of systemic toxicity. Barrington et al. ${ }^{1}$ reported an incidence of systemic anesthetic toxicity to be 0.98 per 1,000 blocks. Our anxiety about the risk of systemic toxicity to local anesthetics has been reduced in recent years by the introduction of the "lipid sink" idea. Prior to this innovation, serious systemic toxic reaction to long-acting local anesthetic drugs was associated with a very poor patient outcome.

With the advent of ultrasound-guided regional anesthesia, experts in regional anesthesia have become aware and concerned about the emerging evidence that intraneural injection is a common occurrence during performance of regional anesthesia. ${ }^{2}$ In addition, studies have demonstrated that commonly used local anesthetics can cause injury to Schwann cells, disrupt the blood-nerve barrier, decrease neural blood flow with associated ischemia, and disrupt cell membrane integrity. ${ }^{3,4}$ Fortunately, the longterm clinical significance of neurotoxicity without direct needle trauma remains rare. Nevertheless, any drug deposited in the perineural region must be clearly evaluated for its local neurotoxicity.

Dextromethorphan has been used as a cough suppressant for decades. Both the parent drug and its metabolite are voltage-dependent sodium channel antagonists, equating them in most ways with local anesthetics. Their local anesthetic properties have been demonstrated in various regional anesthesia techniques, including cutaneous infiltration, ${ }^{5}$ peripheral nerve blockade, ${ }^{6}$ and intrathecal anesthesia. ${ }^{7}$ In this issue of the Journal, Chen et al. ${ }^{8}$ undertook an intriguing step to determine and evaluate the systemic toxicity in rodents of dextromethorphan and its metabolite, dextrorphan. After demonstrating the local anesthetic properties of these two agents in their animal models, they administered equipotent doses of these new 
local anesthetics in the rat models and used bupivacaine in equipotent doses as a control.

Chen et al. ${ }^{8}$ conducted their experiment in two parts. First they evaluated the efficacy of subcutaneous injections of these drugs using the cutaneous trunci muscle reflex. In the second part of the experiment, they studied the systemic effects of these drugs by administering them intravenously. They developed a well-designed in vivo study using a rodent model for the infiltrative cutaneous anesthesia testing. They ensured continuous measurement of objective cardiovascular parameters with the use of sophisticated equipment positioned around the root of the ascending aorta. They employed clearly defined end-points indicating respiratory and cardiac arrest, and the results showed a slight delay of $25-30 \%$, sufficient time for intravenous dextromethorphan and dextrorphan to produce systemic toxicity when compared with bupivacaine.

Although this study and body of research has the undeniable merit of offering valuable insights into systemic toxicity of new compounds for use as local anesthetics, the question remains unanswered regarding their suitability for use in clinical practice. This issue is more relevant now than ever given the advances in technology, such as ultrasound, and evidence that treatment of systemic toxicity with intralipid ${ }^{\circledR}$ (Fresenius Kabi, Uppsala, Sweden) has greatly lessened the threat of mortality from the disorder. In addition, it is important to emphasize that methodological problems in the research design limit our interpretations of its clinical merit. The difficulty of extrapolating data from animals to humans is well recognized, especially in terms of toxic effects of any chemicals. The existence of inter-species differences is undeniable in both pharmacokinetic and pharmacodynamic profiles. This is due to the different disposition and biotransformation of the chemicals as well as the distinct physiological response and sensitivity of species to the chemicals. ${ }^{9}$ Another factor hindering generalizability is the use of a single rodent species for all the studies published on the compounds reviewed. Evaluation of the safety profile of these compounds is of paramount importance. What additional benefits do these new compounds offer in the field of regional anesthesia? We already have access to local anesthetics (ropivacaine and levo-bupivacaine) that are clinically safer, and we now have a reliable treatment for systemic toxicity to local anesthetics. Therefore, it would be difficult ethically to justify testing any new chemical compounds on humans unless the benefits greatly outweigh the risks.

Over the years, a number of compounds with chemical structures mimicking local anesthetics have been tested, but the results have proved disappointing when tested in humans. A good example can be found in the testing of the tricyclic antidepressants (TCAs). Initial testing in animals suggested that we had discovered the "ideal" local anesthetic. The prolonged duration of action of TCAs would obviate the need for continuous catheter techniques and accompanying problems associated with insertion and maintenance. However, the superior potency of TCAs compared with bupivacaine did not prove to be true in human studies. ${ }^{10}$ Furthermore, histological studies in the rodent models demonstrated very serious toxic effects on many tissues, including muscle and nerves, thereby eliminating its potential use as a local anesthetic. ${ }^{11}$ Other examples of failure in this regard include benzyl alcohol and diphenhydramine. Benzyl alcohol has been used as an intradermal anesthetic for intravenous cannulation. Prohibitive toxic effects ${ }^{12-14}$ have made these compounds unlikely clinical candidates. Diphenhydramine shared a similar fate owing to its reported toxic effects ranging from skin necrosis ${ }^{15}$ to a gangrenous extremity warranting amputation. $^{16}$

Our concept of the "ideal" local anesthetic is one that can interact selectively with the voltage-dependent $\mathrm{Na}^{+}$ channels in the peripheral nervous system without any affinity for those in the myocardium or central nervous system. Saxitoxin is one of the few compounds that come close to having these properties. It modulates the outer vestibule of the $\mathrm{Na}^{+}$channel receptors and has selective affinity to certain subgroups that are tissue specific. Unlike bupivacaine, saxitoxin and its analogue, neosaxitoxin, have a very low affinity to the $\mathrm{Na}^{+}$channel subtypes possessed by the cardiac Purkinje fibres. Being more potent than both lidocaine and bupivacaine without myotoxicity or neurotoxicity in vitro or in vivo, ${ }^{17,18}$ its efficacy as a local anesthetic in humans was examined via subcutaneous infiltration on ten healthy volunteers. ${ }^{19}$ Promising results were detected with no adverse effects. This outcome quickly prompted further exploration of prolonging its duration with a sustained-release vehicle, such as liposomal formulations. $^{20}$ The efficacy of saxitoxin as a local anesthetic sounds too good to be true and one must not be overly enthusiastic. The preliminary results were based on a small group of healthy individuals, and there exists a relatively narrow therapeutic index. Furthermore, the limits of saxitoxin on humans have not been thoroughly scrutinized. With the liposomal vesicles, a high concentration of drugs within the carrying vehicle means any margin of error is unforgiving, since it could lead to potentially undefendable catastrophic toxic effects. Thus far, there is no antidote for saxitoxin, although one could weakly argue that diaphragmatic and phrenic nerve palsy responds well to supportive ventilatory treatment. Who knows exactly what other toxic effects a supra-normal dose could have on humans?

At least two other compounds that have local anesthetic and opioid properties, i.e., meperidine and sameridine, 
have been tested. Both have also been successfully used intrathecally to produce surgical anesthesia. Wagner et al. evaluated the local anesthetic properties of meperidine, ${ }^{21}$ showing its ability to inhibit the propagation of action potentials comparable with lidocaine with a fivefold reduced potency. Nonetheless, this reduction in potency has minimal significance in clinical practice. A handful of cases of transient neurological symptoms have been described following subarachnoid injections of meperidine. $^{22,23}$ Needless to say, there has not been a great interest in further clinical use of intrathecal meperidine.

Sameridine is another compound with both opioid and local anesthetic properties. Its potential as an intrathecal local anesthetic was tested in humans in a multicentre trial. ${ }^{24}$ However, this compound was never produced commercially because it did not have any real advantages over currently used local anesthetics.

At present, our existing selection of local anesthetics has been used successfully to induce reversible peripheral nerve blockade. Therefore, for a new drug to compete successfully with the current selection of available local anesthetic drugs, it must demonstrate superior blocking properties and be devoid of undesirable side effects and toxicities. Chen et al. ${ }^{8}$ remind us of the challenges facing investigators on one small aspect of introducing a new drug into the local anesthetic arena. The ultimate drive of the process is cost effectiveness. Drug development requires mammoth investment from pharmaceutical companies. It is estimated that the cost of developing a single new drug can cost $\$ 800$ million US dollars. ${ }^{25}$ Furthermore, if a drug under review fails to meet the stringent safety and toxicity requirements, money invested for unsuccessful compounds is lost and cannot be recovered. This explains the cautious interpretation of results, particularly regarding the translation of animal studies to the human population and the need for a dramatic clinical benefit. The long-term effects of such novel drugs in humans will not be immediately apparent; hence, the existence of regulatory bodies, such as the Food and Drug Administration and the Health Protection Branch, is required to govern the rigorous process and continual evaluation of new drugs on the market. Any clinical use prior to such detailed regulatory evaluation is not recommended.

One hundred and sixteen years have passed since Koller's remarkable discovery demonstrating the local anesthetic properties of cocaine. Within 25 years, the first synthetic local anesthetic was produced (procaine). Since that time, hundreds of compounds with potential local anesthetic properties have been tested, and very few have met the acid test. The search for the "ideal" local anesthetic is a long and painstaking journey, a journey during which most aspirants who set out perish in the attempt. The demonstration by Chen $e t a l .{ }^{8}$ (i.e., showing the lower risk of cardiovascular toxicity from dextromethorphan and its metabolite, dextrorphan, compared with bupivacaine) is important; however, it is not a license for the ad hoc use of these compounds as a clinical local anesthetic. Their work should be seen as a small but solid step on the long journey in search of the "ideal" local anesthetic.

\section{Innover sans toxicité: à la recherche d'un anesthésique local plus sécuritaire}

Depuis plus d'un siècle, la recherche d'un anesthésique local 'idéal' pour l'anesthésie et l'analgésie périopératoire se poursuit. L'anesthésique local idéal sera un agent doté d'un délai d'action rapide aussi bien que d'une durée d'action appropriée et sera capable de bloquer, de façon sélective et réversible, la conduction nerveuse sensitive, mais sans risque de toxicité locale (neurotoxique et myotoxique) ou systémique (neurologique et cardiovasculaire). Depuis que Koller a introduit, en 1884, la cocaïne comme anesthésique en chirurgie, des centaines de molécules ont été testées et ont révélé des effets anesthésiques locaux. Ces agents possèdent différentes structures chimiques: il y a notamment les amino-esters (analogues de la procaïne), les amino-amides (analogues de la lidocaïne), les alcools (alcool benzylique) et le phénol. Toutefois, seuls quelques-uns de ces agents ont une véritable valeur clinique, et la recherche de l'anesthésique local 'idéal' continue.

Pendant des dizaines d'années, la toxicité systémique des anesthésiques locaux a fait l'objet d'un intérêt considérable, en raison surtout des quantités relativement importantes d'anesthésique local administrées pour l'anesthésie régionale. Grâce à l'arrivée de l'anesthésie régionale échoguidée, il est dorénavant possible de déposer très précisément l'anesthésique local à proximité de la structure neurologique désirée. Par conséquent, la plupart des experts de l'anesthésie régionale peuvent produire les effets désirés à l'aide de quantités considérablement réduites d'anesthésiques locaux. De plus, puisqu'il est possible de visualiser l'environnement anatomique de façon dynamique, la diffusion de l'anesthésique local se fait désormais avec un risque nettement réduit d'injection intravasculaire accidentelle. Bien que les données probantes doivent encore voir le jour pour confirmer cette hypothèse, il est probable que ce progrès technologique tienne sa promesse et réduise l'incidence de toxicité systémique. Barrington et coll. ${ }^{1}$ ont rapporté une incidence de toxicité anesthésique systémique de 0,98 par 1000 blocs. Nos inquiétudes quant au risque de toxicité systémique provoquée par les anesthésiques locaux ont diminué ces dernières années grâce à l'introduction du concept 
de «siphon lipidique». Avant cette innovation, toute réaction toxique systémique grave à des anesthésiques locaux à action prolongée était associée à un pronostic sombre pour le patient.

Avec l'apparition de l'anesthésie régionale échoguidée, les experts de l'anesthésie régionale ont réalisé qu'un nombre croissant de données probantes indiquaient que l'injection intraneurale était un événement courant pendant l'anesthésie régionale, et s'en sont inquiétés. ${ }^{2}$ De plus, des études ont montré que les anesthésiques fréquemment utilisés pouvaient provoquer des lésions au niveau des cellules de Schwann, rompre la barrière sang-nerf, réduire le débit sanguin neural et ainsi favoriser l'ischémie associée, et rompre l'intégrité de la membrane cellulaire., Heureusement, l'importance clinique à long terme de la neurotoxicité sans traumatisme direct lié à l'aiguille reste rare. Il n'en demeure pas moins que la neurotoxicité locale de tout médicament déposé dans la région périnerveuse doit être clairement évaluée.

Le dextrométhorphane est utilisé comme antitussif depuis des dizaines d'années. La molécule mère et son métabolite sont tous deux des antagonistes des canaux sodiques voltage-dépendants, ce qui les rend quasi équivalents aux anesthésiques locaux. Leurs propriétés anesthésiques locales ont été démontrées dans plusieurs techniques d'anesthésie régionale, notamment les infiltrations cutanées, ${ }^{5}$ les blocs des nerfs périphériques ${ }^{6}$ et l'anesthésie intrathécale. ${ }^{7}$ Dans ce numéro du Journal, Chen et coll. ${ }^{8}$ ont entrepris, de façon intrigante, de déterminer et d'évaluer la toxicité systémique du dextrométhorphane et de son métabolite, le dextrorphane, chez le rongeur. Après avoir démontré les propriétés d'anesthésique local de ces deux agents dans leurs modèles animaux, les auteurs ont administré des doses équipotentes de ces nouveaux anesthésiques locaux dans leur modèle de rats et ont utilisé la bupivacaïne à des doses équipotentes comme traitement de référence.

Chen et coll. ${ }^{8}$ ont réalisé leur expérience en deux parties. Ils ont d'abord évalué l'efficacité des injections sous-cutanées de ces médicaments en mesurant le réflexe du muscle cutané du tronc. Dans la deuxième partie de l'expérience, ils ont étudié les effets systémiques des médicaments en les administrant par voie intraveineuse. Ils ont mis au point une étude in vivo bien conçue à dans un modèle de rongeur pour réaliser des tests d'anesthésie cutanée par infiltration. En utilisant des équipements de pointe placés autour de la racine de l'aorte ascendante, les auteurs ont pu garantir des mesures continues de paramètres cardiovasculaires objectifs. Ils ont employé des critères d'évaluation clairement définis indiquant l'arrêt respiratoire et cardiaque, et les résultats ont révélé un léger retard de 25-30\% pour que le dextrométhorphane et le dextrorphane provoquent une toxicité systémique lorsque ces agents étaient comparés à la bupivacaïne.

Bien que cette étude et ce corpus de recherche aient le mérite incontesté de nous offrir d'intéressantes perspectives quant à la toxicité systémique des nouvelles molécules qui pourraient être utilisées comme anesthésiques locaux, la question de savoir si elles sont adaptées à une utilisation dans la pratique clinique demeure sans réponse. Cette question est plus pertinente que jamais étant donné les progrès technologiques tels l'échoguidage, sans oublier les données probantes appuyant qu'un traitement de la toxicité systémique à l'aide d'intralipide ${ }^{\circledR}$ (Fresenius Kabi, Uppsala, Suède) réduisait considérablement la menace de mortalité liée à ce trouble. De plus, il convient de souligner que certains problèmes méthodologiques dans la conception de l'étude limitent notre appréciation de ses mérites cliniques. La difficulté liée à l'extrapolation de données d'études animales vers l'humain est bien connue, particulièrement quand il s'agit des effets toxiques d'agents chimiques. L'existence de différences entre les espèces est indéniable, tant au niveau des profils pharmacocinétiques que pharmacodynamiques. Ceci est dû aux différences dans la disposition et la biotransformation des agents chimiques ainsi qu'à la réponse physiologique et à la sensibilité distinctes d'une espèce donnée aux agents chimiques. ${ }^{9}$ Un autre facteur entravant l'aspect généralisable de telles réactions est l'utilisation d'une seule espèce de rongeur dans toutes les études publiées sur les molécules à l'étude. L'évaluation du profil d'innocuité de ces molécules est cruciale. Quels sont les avantages supplémentaires offerts par ces nouvelles molécules dans le domaine de l'anesthésie régionale? Nous avons déjà accès à des anesthésiques locaux (la ropivacaïne et la lévo-bupivacaïne) qui sont plus sécuritaires d'un point de vue clinique, et nous disposons aujourd'hui d'un traitement fiable pour prendre en charge la toxicité systémique aux anesthésiques locaux. Par conséquent, il serait difficile, d'un point de vue éthique, de justifier la réalisation d'essais avec de nouveaux composés chimiques chez l'humain, à moins que les bienfaits l'emportent clairement sur les risques.

$\mathrm{Au}$ fil des ans, plusieurs molécules possédant des structures chimiques semblables à celles des anesthésiques locaux ont été testées, mais les résultats se sont avérés décevants lorsqu'ils étaient testés chez l'humain. Les tests réalisés avec les antidépresseurs tricycliques (ATC) en sont un bon exemple. Les premiers tests sur les animaux suggéraient la découverte de l'anesthésique local 'idéal'. La durée d'action prolongée des ATC écarterait le besoin de techniques de cathéters continus et les problèmes associés d'insertion et de maintien. Toutefois, la puissance supérieure des ATC par rapport à la bupivacaïne ne s'est pas confirmée dans les études chez l'humain. ${ }^{10}$ De plus, les 
études histologiques réalisées sur des modèles de rongeurs ont révélé des effets toxiques très graves sur de nombreux tissus, y compris les muscles et les nerfs, éliminant ainsi leur potentiel d'utilisation en tant qu'anesthésique local. ${ }^{11}$ L'alcool benzylique et la diphenhydramine sont d'autres exemples d'échecs similaires. L'alcool benzylique est utilisé comme anesthésique intradermique pour la canulation intraveineuse. Les effets toxiques prohibitifs ${ }^{12-14}$ de ces molécules en ont fait des candidats cliniques peu probables. La diphenhydramine a partagé le même sort en raison de ses effets toxiques rapportés, allant de la nécrose cutanée $^{15}$ à un membre gangrené nécessitant l'amputation. ${ }^{16}$

Notre idée de l'anesthésique local 'idéal' est celle d'un anesthésique qui puisse interagir de façon sélective avec les canaux sodiques voltage-dépendants dans le système nerveux périphérique et ce, sans présenter d'affinité pour les canaux situés dans le myocarde ou le système nerveux central. La saxitoxine est l'une des rares substances à être dotée de ces propriétés. Elle module le vestibule externe des récepteurs des canaux $\mathrm{Na}^{+}$et possède une affinité sélective pour certains sous-groupes spécifiques à certains tissus. À la différence de la bupivacaïne, la saxitoxine et son analogue, la néosaxitoxine, ont une très faible affinité pour les sous-types des canaux $\mathrm{Na}^{+}$des fibres de Purkinje au niveau du coeur. Étant plus puissante que la lidocaïne et que la bupivacaïne sans myotoxicité ou neurotoxicité in vitro ou in vivo, ${ }^{17,18}$ son efficacité comme anesthésique local a été examinée chez l'humain par infiltration sous-cutanée chez dix volontaires sains. ${ }^{19}$ Des résultats prometteurs ont été dépistés sans effets indésirables. Ce résultat a rapidement mené à une exploration plus approfondie des façons de prolonger son action à l'aide d'un véhicule à libération soutenue, notamment les formules liposomales. ${ }^{20}$ L'efficacité de la saxitoxine en tant qu' anesthésique local semble trop belle pour être vraie; c'est pourquoi il ne faut pas crier victoire trop vite. Les résultats préliminaires s'appuyaient sur un petit groupe de volontaires sains, et l'indice thérapeutique est relativement faible. En outre, nous n'avons pas encore examiné en profondeur les limites de la saxitoxine chez l'humain. Avec les vésicules liposomales, une concentration élevée de médicament dans le véhicule signifie qu'aucune marge d'erreur n'est possible, étant donné que cela pourrait provoquer des effets toxiques catastrophiques potentiellement indéfendables. À ce jour, il n'existe pas d'antidote à la saxitoxine, bien qu'on puisse avancer que la paralysie diaphragmatique et du nerf phrénique réponde bien au traitement respiratoire de soutien - mais cet argument est faible. Qui sait exactement quels autres effets toxiques une dose supérieure à la normale pourrait avoir sur l'humain?
Au moins deux autres molécules possédant des propriétés anesthésiques locales et opioïdes, soit la mépéridine et la saméridine, ont été testées. Ces deux agents ont été utilisés avec succès par voie intrathécale pour réaliser une anesthésie chirurgicale. Wagner et coll. ont évalué les propriétés anesthésiques locales de la mépéridine ${ }^{21}$ et ont montré que sa capacité à inhiber la propagation des potentiels d'action était comparable à celle de la lidocaïne avec une puissance réduite de cinq fois. Toutefois, cette puissance réduite n'a qu'un impact minime sur la pratique clinique. Quelques cas de symptômes neurologiques transitoires ont été décrits après des injections sous-arachnoïdiennes de mépéridine. ${ }^{22,23}$ Inutile de préciser que l'intérêt pour une utilisation clinique plus poussée de la mépéridine intrathécale n'a par la suite pas été très soutenu.

La saméridine est une autre substance possédant des propriétés opioïdes et anesthésiques locales. Son potentiel en tant qu'anesthésique local intrathécal a été testé chez l'humain dans une étude multicentrique. ${ }^{24}$ Cependant, cette molécule n'a jamais été produite de façon commerciale parce qu'elle ne présentait pas d'avantages réels comparativement aux anesthésiques locaux actuellement disponibles.

À ce jour, l'éventail d'anesthésiques locaux existant a été utilisé avec succès pour induire des blocs réversibles des nerfs périphériques. Par conséquent, si un nouveau médicament veut l'emporter sur l'éventail actuellement disponible d'anesthésiques locaux, il doit posséder des propriétés de blocage supérieures et ne pas présenter d'effets secondaires indésirables ou de toxicités. Chen et coll. ${ }^{8}$ nous rappellent les défis auxquels font face les chercheurs en ce qui touche à un aspect mineur de l'introduction d'un nouveau médicament sur le marché des anesthésiques locaux: la motivation de ce processus est, en fin de compte, la rentabilité. La mise au point d'un médicament nécessite des investissements gigantesques de la part des compagnies pharmaceutiques. On estime que le coût de mise au point d'un seul nouveau médicament peut s'élever à 800 millions de dollars US. ${ }^{25}$ De plus, si un médicament à l'étude n'est pas conforme aux exigences rigoureuses d'innocuité et de toxicité, l'argent investi dans des composés sans avenir est perdu et ne peut être récupéré. Ceci explique l'interprétation prudente des résultats, particulièrement concernant la traduction d'études animales vers la population humaine et le besoin d'un avantage clinique sans équivoque. Les effets à long terme de ces nouveaux médicaments chez l'humain ne seront pas immédiatement visibles, d'où l'existence d'organismes de réglementation tels que la Food and Drug Administration américaine et la Direction générale de la protection de la santé, qui sont nécessaires pour régir le 
processus rigoureux et l'évaluation continue des nouveaux médicaments sur le marché. Toute utilisation clinique survenant avant une telle évaluation réglementaire détaillée n'est pas recommandée.

Cent seize ans se sont écoulés depuis la découverte remarquable de Koller démontrant les propriétés anesthésiques locales de la cocaïne. Dans les 25 premières années suivant cette découverte, le premier anesthésique local de synthèse a vu le jour (la procaïne). Depuis, des centaines de substances qui pouvaient posséder des propriétés anesthésiques locales ont été testées, et seuls quelques-unes ont passé le test de l'acide. La recherche de l'anesthésique local 'idéal' est un voyage à la fois long et pénible, un voyage pendant lequel la plupart des aspirants présents à la ligne de départ périront. La démonstration de Chen et coll. ${ }^{8}$ (c.-à-d. de démontrer le risque réduit de toxicité cardiovasculaire du dextrométhorphane et de son métabolite, le dextrorphane, par rapport à la bupivacaïne) est importante; toutefois, elle n'autorise pas l'utilisation ad hoc de ces médicaments comme anesthésiques locaux en clinique. Leurs travaux devraient être interprétés comme un pas, à la fois petit et bien ancré, sur la longue route à la recherche de l'anesthésique local 'idéal'.

Acknowledgements We are indebted to Drs. Brendan Finucane and Saifudin Rashiq, Department of Anesthesiology at the University of Alberta for their invaluable comments and suggestions.

Conflicts of interest None declared.

\section{References}

1. Barrington MJ, Watts SA, Gledhill SR, et al. Preliminary results of the Australasian Regional Anaesthesia Collaboration: a prospective audit of more than 7000 peripheral nerve and plexus blocks for neurologic and other complications. Reg Anesth Pain Med 2009; 34: 534-41.

2. Sala Blanch $X$, Lopez AM, Carazo J, et al. Intraneural injection during nerve stimulator-guided sciatic nerve block at the popliteal fossa. Br J Anaesth 2009; 102: 855-61.

3. Kalichman $M W$. Physiologic mechanisms by which local anesthetics may cause injury to nerve and spinal cord. Reg Anesth 1993; 18: 448-52.

4. Kitagawa N, Oda M, Totoki T. Possible mechanism of irreversible nerve injury caused by local anesthetics: detergent properties of local anesthetics and membrane disruption. Anesthesiology 2004; 100: 962-7.

5. Chen YW, Chu KS, Lin CN, et al. Dextromethorphan or dextrorphan have a local anesthetic effect on infiltrative cutaneous analgesia in rats. Anesth Analg 2007; 104: 1251-5.
6. Hou CH, Tzeng JI, Chen YW, et al. Dextromethorphan, 3-methoxymorphinan, and dextrorphan have local anaesthetic effect on sciatic nerve blockade in rats. Eur J Pharmacol 2006; 544: 10-6.

7. Chen $Y W$, Chen YC, Lin CN, et al. The spinal anaesthetic effect of dextromethorphan, dextrorphan, and 3-methoxymorphinan. Eur J Pharmacol 2007; 569: 188-93.

8. Chen YW, Wang JJ, Liu TY, Chen YC, Hung CH. Systemic dextromethorphan and dextrorphan are less toxic in rats than bupivacaine at equianesthetic doses. Can J Anesth 2011; 58. DOI: 10.1007/s12630-010-9408-z.

9. Garattini $S$. Toxic effects of chemicals: difficulties in extrapolating data from animals to man. Crit Rev Toxicol 1985; 16: 1-29.

10. Fridrich $P$, Eappen $S$, Jaeger $W$, et al. Phase Ia and Ib study of amitriptyline for ulnar nerve block in humans: side effects and efficacy. Anesthesiology 2004; 100: 1511-8.

11. Barnet CS, Louis DN, Kohane DS. Tissue injury from tricyclic antidepressants used as local anesthetics. Anesth Analg 2005; 101: 1838-43.

12. Hetherington NJ, Dooley MJ. Potential for patient harm from intrathecal administration of preserved solutions. Med J Aust 2000; 173: 141-3.

13. Hiller JL, Benda GI, Rahatzad M, et al. Benzyl alcohol toxicity: impact on mortality and intraventricular hemorrhage among very low birth weight infants. Pediatrics 1986; 77: 500-6.

14. Menon PA, Thach BT, Smith CH, et al. Benzyl alcohol toxicity in a neonatal intensive care unit. Incidence, symptomatology, and mortality. Am J Perinatol 1984; 1: 288-92.

15. Howard K, Conrad T, Heiser J, Manzi JA. Diphenhydramine hydrochloride as a local anesthetic. A case report. J Am Podiatry Assoc 1984; 74: 240-2.

16. Ramsdell WM. Severe reaction to diphenhydramine. J Am Acad Dermatol 1989; 21: 1318-20.

17. Kohane DS, Lu NT, Gokgol-Kline AC, et al. The local anesthetic properties and toxicity of saxitonin homologues for rat sciatic nerve block in vivo. Reg Anesth Pain Med 2000; 25: 52-9.

18. Sakura S, Bollen AW, Ciriales $R$, Drasner $K$. Local anesthetic neurotoxicity does not result from blockade of voltage-gated sodium channels. Anesth Analg 1995; 81: 338-46.

19. Rodriguez-Navarro AJ, Lagos $N$, Lagos $M$, et al. Neosaxitoxin as a local anesthetic: preliminary observations from a first human trial. Anesthesiology 2007; 106: 339-45.

20. Epstein-Barash H, Shichor I, Kwon AH, et al. Prolonged duration local anesthesia with minimal toxicity. Proc Natl Acad Sci USA 2009; 106: 7125-30.

21. Wagner LE 2nd, Eaton M, Sabnis SS, Gingrich KJ. Meperidine and lidocaine block of recombinant voltage-dependent $\mathrm{Na}+$ channels: evidence that meperidine is a local anesthetic. Anesthesiology 1999; 91: 1481-90.

22. Lewis WR, Perrino AC Jr. Transient neurological symptoms after subarachnoid meperidine. Anesth Analg 2002; 94: 213-4.

23. Urmey WF. Spinal anaesthesia for outpatient surgery. Best Pract Res Clin Anaesthesiol 2003; 17: 335-46.

24. Mulroy MF, Greengrass $R$, Ganapathy $S$, Chan V, Heierson A. Sameridine is safe and effective for spinal anesthesia: a comparative dose-ranging study with lidocaine for inguinal hernia repair. Anesth Analg 1999; 88: 815-21.

25. Roberts $T G J r$, Lynch $T J J r$, Chabner BA. The phase III trial in the era of targeted therapy: unraveling the "go or no go" decision. J Clin Oncol 2003; 21: 3683-95. 